\title{
Online Fault Diagnosis of Air-conditioning Units Using Recurrent Neural Network Trained with Simulation Data
}

\author{
Upali Samarasinghe Herath Kumarage* and Shuji Hashimoto*
}

\begin{abstract}
Fault detection and diagnosis (FDD) of air-conditioning systems is very important for energy saving, safety and maintenance and in high demand. The air-conditioning system is a complex and dynamic system which makes the diagnosis difficult because the observed parameters do not directly express the state of the system. On the other hand, faults analysis and fault cases collected from the actual air-conditioning system are not practicable. With due consideration of the above points, the authors propose an effective FDD method for air-conditioning systems using model simulation and Recurrent Neural Network (RNN). An air-conditioning system of a certain building is used as a model in this research. The validity of the model is confirmed by comparing the simulation data with real observations. In this study, the authors concentrated on several typical faults in both chilled and hot water volume control valves. The authors obtained the data of the normal and some fault cases in time sequence using the model simulation. As a result of the current experiments, the recognition rate was over $90 \%$ and it was demonstrated that the proposed method is suitable for practical use.
\end{abstract}

Key Words : air-conditioning system, model simulation, recurrent neural network, fault detection and diagnosis

\section{Introduction}

Air-conditioning systems for buildings have become increasingly diversified and complicated year by year and the fault detection and diagnosis (FDD) of these air-conditioning systems has recently been in high demand. The FDD of air-conditioning systems is important not only for warranty of habitability but also for the maintenance and the higher-level of reliability and availability of the system as well as energy saving etc. Although a variety of FDD methods have been proposed ${ }^{1) \sim 6)}$ so far, but there is no established online FDD method because of the following factors of difficulty:

1. Nonlinear characteristic: The air-conditioning system displays nonlinear characteristics. and it requires a FDD method, which is adaptable to nonlinear characteristic behavior.

2. Dynamic behavior: The air-conditioning system displays complex dynamic behavior, which makes the diagnosis difficult.

3. Indirectness of the information: The observable parameters of the air-conditioning system are not directly expressing the state of the system.

4. Unknown fault patterns : In order to detect or diagnose faults online, most of the FDD methods require the

\footnotetext{
* Department of Applied Physics, Waseda University,

3-4-1 Okubo, Shinjuku-ku, Tokyo

(Received January 5, 2007)

(Revised November 3, 2007)
}

fault patterns in advance. But obtaining the fault patterns in an active system is very difficult, since it would normally be unacceptable to generate the faults in an occupied building and that some situations are difficult to reproduce.

In this research, the authors proposed an online fault diagnosis system using Recurrent Neural Network (RNN). In this case, sequential data on normal and fault cases of the air-conditioning system is required. But usually a broad range of those data is not available and practically they cannot be obtained from the actual system. To solve this, the authors developed a thermodynamic simulation model for generating those data, instead of using the actual system. This is also an advantage of this research.

The authors applied the RNN as a solution of the above-mentioned problems 1,2 and 3 . The RNN is a type of neural network that has feedback connections. In principle, it can implement almost arbitrary sequential behavior, which makes them promising for nonlinear dynamic applications. Moreover, the RNN is biologically more plausible and computationally more powerful than other adaptive models such as Hidden Markov Models (with no continuous internal states), feed forward networks and Support Vector Machines (with no internal states at all). Therefore they are not suitable for continuous time series analyses. The authors introduced a model simulation to solve the problem of the difficulty 4 . The thermodynamic computer model simulation could be 
developed according to the details, information, and the measurements obtained from the building. Consequently, fault patterns over various assumed conditions were obtained and a fault patterns database for aiding the FDD was created.

This paper is structured as follows. Section 2 describes the air-conditioning system of a specific office building, its model simulation and evaluation results of the simulation experiments. A fault database generating method is introduced in Section 3. The applied FDD method and experimental results are described in Sections 4 and 5 . The conclusions and future challenges are given in Section 6.

\section{Model Simulation}

Many simulations have been verified by comparison with measured results from the actual building in both winter (heating) and summer (cooling) over a wide range of weather conditions and setting status. These simulations cover a range of an air-conditioning system performance parameters and regional climate conditions. These models do not explicitly include any moisture transport phenomena. This is mainly due to the lack of verified models of moisture performance for buildings in general and specifically, those systems do not control the humidity and can be used based on the simple information available for office buildings.

\subsection{Referenced Air-conditioning System}

The variable air volume (VAV) system using chilled and hot water equipped in an occupied specific office building was employed in this research. The VAV system is shown in Fig. 1.

The air-conditioning system consists of dampers, fans, a cooling unit, a heating unit, a humidifying unit, and their controllers. In heating, the supply air temperature is controlled by hot water in the heating unit. In cooling, the temperature and the humidity of the supply air are controlled by chilled and hot water. First, the air is cooled down and the moisture is eliminated in chilled water in the cooling unit. Next, it is warmed up by hot water in the heating unit. Lastly, the air is humidified by chilled water in the humidify unit. Chilled and the hot water valves are controlled by the velocity proportional-integral-derivative (PID), controlling method expressed in Eq. ( 1 ). The chilled water valve is adjusted by on-off control. For the opening state of the chilled and hot water control valves, $100 \%$ represents the full opening while $0 \%$ represents the full closing. The temperature of chilled water is kept constant at $12^{\circ} \mathrm{C}$ and the temperature of hot water is set to $60^{\circ} \mathrm{C}$.

$$
\begin{aligned}
u(t)= & K_{P}\{e(t)-e(t-1)\}+K_{I} e(t) \\
& +K_{D}\{e(t)-2 e(t-1)+e(t-2)\}
\end{aligned}
$$

$u(t)$ : Control variable value at time $t$

$e(t)$ : Difference between setting and actual temperatures of the room at time $t$

$K_{P}$ : Proportional Gain, $K_{I}$ : Integral Gain, $K_{D}$ : Derivative Gain

\section{2 Heat Transfer Calculations}

The authors used a room ( 280 cubic meters) on the 3 rd floor of the building B at the Robot Plaza in Gifu prefecture. For heat transfer calculation, the authors monitored temperature and relative humidity at 30 -second interval at several locations given in Table 1. The authors also referred to the construction materials, dimensions and orientation of the building, number of people in the airconditioned area, office automation equipment, and areas of the walls, windows, floor, ceiling, the draft of the room and the air-conditioning handbook ${ }^{7)}$ for regional solar

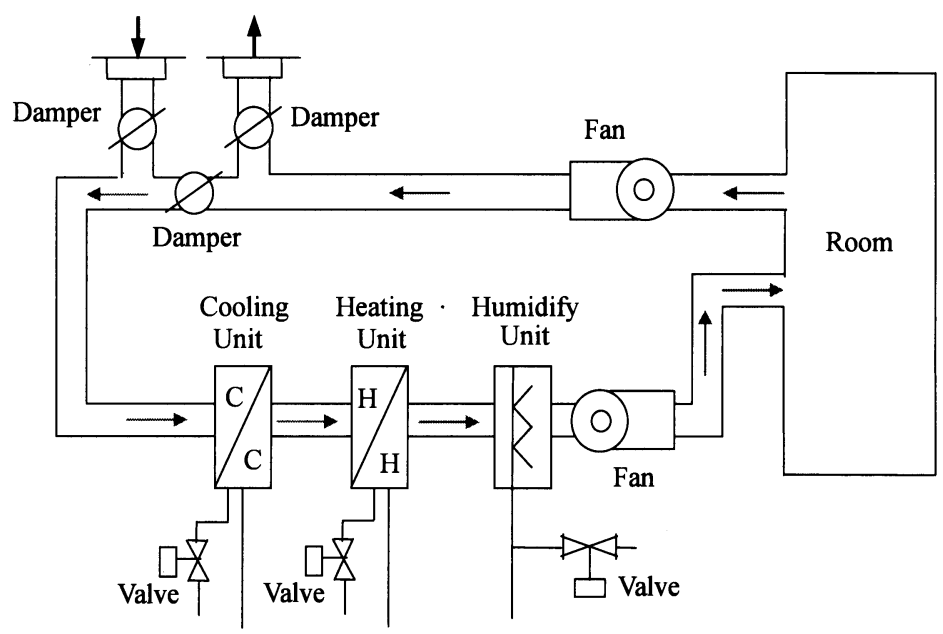

Fig. 1 Referenced air-conditioning system 
Table 1 Data monitored locations

\begin{tabular}{|c|c|c|c|}
\hline No & Monitored Location & No & Monitored Location \\
\hline 1 & $3 \mathrm{~F}$ Outdoor temperature (East) & 13 & $3 \mathrm{~F}$ Inside-wall surface temperature (West) \\
\hline 2 & $3 \mathrm{~F}$ Outdoor temperature (West) & 14 & $3 \mathrm{~F}$ Inside-wall surface temperature (South) \\
\hline 3 & 3.F Outdoor temperature (South) & 15 & $3 \mathrm{~F}$ Inside-wall surface temperature (North) \\
\hline 4 & $3 \mathrm{~F}$ Outdoor temperature (North) & 16 & $3 \mathrm{~F}$ Floor surface temperature (North) \\
\hline 5 & $3 \mathrm{~F}$ Outdoor temperature (Roof) & 17 & $3 \mathrm{~F}$ Outdoor surface temperature (Roof) \\
\hline 6 & $3 \mathrm{~F}$ Temperature (Coridoor) & 18 & $3 \mathrm{~F}$ Surface temperature (Coridoor) \\
\hline 7 & 3 F Temperature (Room) & 19 & 3 F Humidity (Room) \\
\hline 8 & $3 \mathrm{~F}$ Outside-wall surface temperature (East) & 20 & 3 F Relative humidity（Roof） \\
\hline 9 & $3 \mathrm{~F}$ Outside-wall surface temperature (West) & 21 & 3 F Relative humidity (Coridoor) \\
\hline 10 & $3 \mathrm{~F}$ Outside-wall surface temperature (South) & 22 & 2 F Temperature (Room) \\
\hline 11 & $3 \mathrm{~F}$ Outside-wall surface temperature (North) & 23 & 2 F Humidity（Room） \\
\hline 12 & $3 \mathrm{~F}$ Inside-wall surface temperature (East) & 24 & 2 F Surface temperature (Ceeling) \\
\hline
\end{tabular}

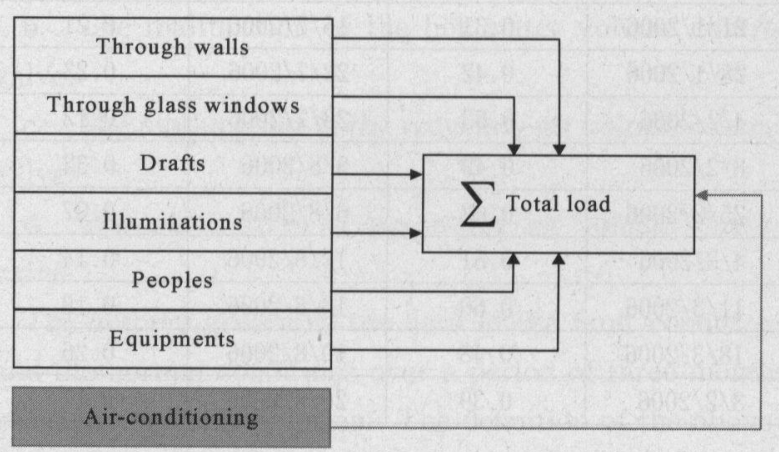

Fig. 2 Heat load calculations factors

radiation information. The heat load calculation process is shown in Fig. 2 and the authors calculated Eq. (1) through Eq.(18) in the model simulations and set the parameters of the simulator given in the nomenclature by referring to the documents provided by the construction company.

\section{Loads through walls}

Outer walls, cooling

$$
Q_{W_{-} \text {out }}=\sum_{d=N}^{S N}\left(T_{\text {out } \_d}-T_{\text {room }}\right) \times K_{w_{-} d} \times A_{w_{-} d}
$$

Outer walls, heating

$$
Q_{W_{-} \text {out }}=\sum_{d=N}^{S N}\left(T_{\text {out } d}-T_{\text {room }}\right) \times K_{w_{-} d} \times A_{w_{-} d} \times k_{d}
$$

Inner walls, cooling and heating

$$
Q_{W_{-} \text {in }}=\sum_{d=N}^{S N}\left(T_{\text {nroomt } \_d}-T_{\text {room }}\right) \times K_{w_{-} d} \times A_{w_{-} d}
$$

\section{Loads through glass windows}

Outer glass windows, cooling

$$
Q_{G_{-} \text {out }}=\sum_{d=N}^{S N}\left(q_{G R_{-} d}+q_{G C_{-} d}\right)
$$

Solar radiation

$$
\begin{aligned}
q_{G R_{-} d}= & \left(\left(S_{G R_{-} d} \times C C_{R}\right)+S_{G P_{-} d} \times\left(1-C C_{R}\right) \times S_{G R L_{-} d}\right) \\
& \times S C_{d} \times A_{g_{-} d}
\end{aligned}
$$

\section{Percolation}

$$
q_{G C_{-} d}=\left(T_{\text {out } \_d}-T_{\text {room }}\right) \times K_{g_{-} d} \times A_{g_{-} d}
$$

Outer glass windows, heating

$$
Q_{G_{-} \text {out }}=\sum_{d=N}^{S N}\left(T_{\text {out } \_d}-T_{\text {room }}\right) \times K_{g_{-} d} \times A_{g_{-} d}
$$

Inner glass windows, cooling and heating

$$
Q_{G_{-} \text {in }}=\sum_{d=N}^{S N}\left(T_{\text {nroom } \_d}-T_{\text {room }}\right) \times K_{g_{-} d} \times A_{g_{-} d}
$$

\section{Loads through roof and floor}

Roof

$$
Q_{\text {roof }}=\left(T_{\text {out_roof }}-T_{\text {room }}\right) \times K_{\text {roof }} \times A_{\text {roof }}
$$

Floor

$$
Q_{\text {floor }}=\left(T_{\text {out_floor }}-T_{\text {room }}\right) \times K_{\text {floor }} \times A_{\text {floor }}
$$

\section{Draft loads}

$$
Q_{D}=q_{D_{-} S}+q_{D_{-} L}
$$

Sensible load

$$
q_{D_{-} s}=\left(T_{\text {out } \_a v}-T_{\text {room }}\right) \times K_{H V} \times V_{D}
$$

Latent load

$$
q_{D_{-} L}=\left(T_{\text {out_av }}-T_{\text {room }}\right) \times K_{W E L H} \times V_{D}
$$

\section{Human body loads}

Sensible load+Latent load

$$
Q_{H}=q_{H S}+q_{H L}
$$

\section{Illumination loads}

Fluorescent bulbs load+Incandescent bulbs load

$$
\begin{aligned}
& Q_{I}=q_{I F}+q_{I I} \\
& q_{I F}=\left(1.0 \times W_{F}+0.5 \times W_{F} \times A M F_{F}\right) \times A_{\text {floor }} \\
& q_{I I}=0.86 \times W_{I} \times A_{\text {floor }}
\end{aligned}
$$

\section{Equipment loads}

The equipment load depends on the equipment used in the room. Therefore, the authors estimated it according to the electrical power consumption.

\section{3 Model Simulations and Evaluation}

Firstly, the authors compared the heat transfer phenomena between the simulator and the actual room without 


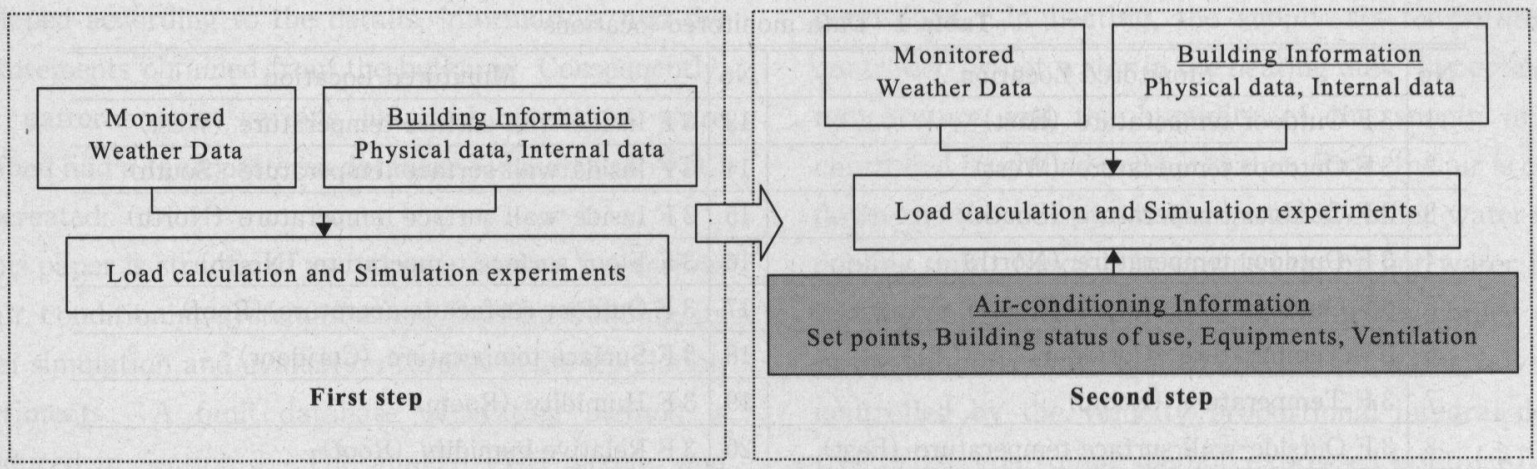

Fig. 3 Simulation Procedure

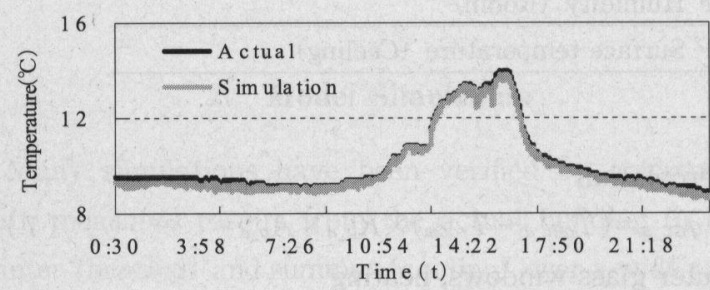

(a) Air-conditioning (OFF)

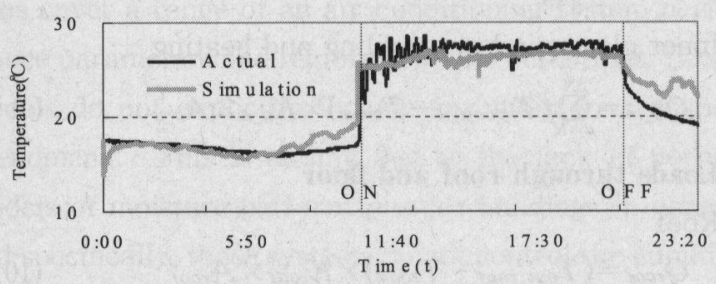

(b) Air-conditioning (ON)

Fig. 4 Simulation result

air-conditioning system for parameter tuning. Secondly, the air-conditioning control was added as shown in Fig. 3. The result of the experiment was evaluated with the mean absolute error as given in Eq. (19), where $S(t), A(t)$ and $N$ represent simulated room temperature, actual room temperature and the number of observation, respectively. The observation was done every 30 seconds.

$$
Y=\frac{1}{N} \sum_{t=1}^{N}|S(t)-A(t)|
$$

Fig. 4 shows the typical temperature profiles with and without the air-conditioning system and the evaluations of these two cases are given in Tables 2 and 3 . In Table 3, there still exist some differences between the simulation and the real data. They were depending on whether the doors and windows are open or closed. It was not possible to know the state of the doors and windows in the occupied buildings. However, the room temperature of each simulation changed as the actual room temperature did. Therefore it is possible to expect that the differences between normal and fault data in the actual system are similar to the simulation results. Meanwhile, the RNN learned the
Table 2 Evaluation result (without AC)

\begin{tabular}{c|c|c|c}
\hline \hline \multicolumn{1}{c|}{ Date } & $\mathrm{Y}$ & Date & $\mathrm{Y}$ \\
\hline $14 / 1 / 2006$ & 0.42 & $8 / 7 / 2006$ & 0.17 \\
\hline $21 / 1 / 2006$ & 0.31 & $15 / 7 / 2006$ & 0.21 \\
\hline $28 / 1 / 2006$ & 0.42 & $22 / 7 / 2006$ & 0.23 \\
\hline $4 / 2 / 2006$ & 0.53 & $23 / 7 / 2006$ & 0.13 \\
\hline $8 / 2 / 2006$ & 0.49 & $5 / 8 / 2006$ & 0.32 \\
\hline $25 / 2 / 2006$ & 0.62 & $6 / 8 / 2006$ & 0.27 \\
\hline $4 / 3 / 2006$ & 0.51 & $12 / 8 / 2006$ & 0.14 \\
\hline $11 / 3 / 2006$ & 0.60 & $13 / 8 / 2006$ & 0.18 \\
\hline $18 / 3 / 2006$ & 0.48 & $19 / 8 / 2006$ & 0.26 \\
\hline $3 / 2 / 2006$ & 0.39 & $26 / 8 / 2006$ & 0.11 \\
\hline
\end{tabular}

Table 3 Evaluation renult (with $\mathrm{AC}$ )

\begin{tabular}{c|c|c|c}
\hline \hline Date & $\mathrm{Y}$ & Date & $\mathrm{Y}$ \\
\hline $17 / 1 / 2006$ & 1.13 & $13 / 2 / 2006$ & 1.05 \\
\hline $18 / 1 / 2006$ & 2.80 & $14 / 2 / 2006$ & 1.89 \\
\hline $19 / 1 / 2006$ & 2.22 & $15 / 2 / 2006$ & 1.31 \\
\hline $26 / 1 / 2006$ & 2.62 & $21 / 2 / 2006$ & 0.65 \\
\hline $27 / 1 / 2006$ & 2.57 & $22 / 2 / 2006$ & 1.61 \\
\hline $28 / 1 / 2006$ & 1.48 & $23 / 2 / 2006$ & 1.02 \\
\hline $31 / 1 / 2006$ & 2.07 & $24 / 2 / 2006$ & 1.59 \\
\hline $1 / 2 / 2006$ & 2.15 & $27 / 2 / 2006$ & 1.51 \\
\hline $2 / 2 / 2006$ & 2.08 & $7 / 7 / 2006$ & 1.32 \\
\hline $3 / 2 / 2006$ & 2.50 & $8 / 7 / 2006$ & 1.55 \\
\hline $4 / 2 / 2006$ & 3.01 & $10 / 7 / 2006$ & 1.79 \\
\hline $6 / 2 / 2006$ & 3.06 & $14 / 7 / 2006$ & 1.24 \\
\hline $7 / 2 / 2006$ & 1.81 & $18 / 7 / 2006$ & 1.84 \\
\hline $9 / 2 / 2006$ & 3.21 & $1 / 8 / 2006$ & 0.96 \\
\hline $10 / 2 / 2006$ & 2.05 & $2 / 8 / 2006$ & 1.86 \\
\hline $11 / 2 / 2006$ & 2.46 & $8 / 8 / 2006$ & 1.44 \\
\hline
\end{tabular}

differences of each normal and fault data set. Accordingly, it can be assumed that the developed simulator is good enough for the fault data generation. 


\section{Generated Faults}

The authors focused their diagnosis on four faults for testing the trend diagnosis. Assuming that certain patterns or symptoms are associated with each fault, the fault diagnosis strategy would analyze the difference between normal and existing patterns. Using the developed simulator, the authors measured the temperatures of the outside, the room, the mixing part of the recycling air, the intake air from outside, the exit of the cooling unit, and the exit of the heating unit under both normal and fault conditions.

The following faults were focused on :

a. The malfunction of the chilled water volume control valve.

b. The malfunction of the hot water volume control valve.

c. The malfunction of the recycling air volume control valve.

d. The malfunction of the intake air volume control valve.

The authors generated the data of the fault conditions and the normal conditions over a period of three months using the model simulation. The definition of the abovementioned four faults of the valves is described in Table 4 with tree cases as the full close of the valve [Full], the

Table 4 Fault definition

\begin{tabular}{c|c|c|c}
\hline \hline Position & Full & Stuck & Response \\
\hline $\mathrm{a}$ & Yes & Yes & Yes \\
\hline $\mathrm{b}$ & Yes & Yes & Yes \\
\hline $\mathrm{c}$ & Yes & No & No \\
\hline $\mathrm{d}$ & Yes & No & No \\
\hline
\end{tabular}

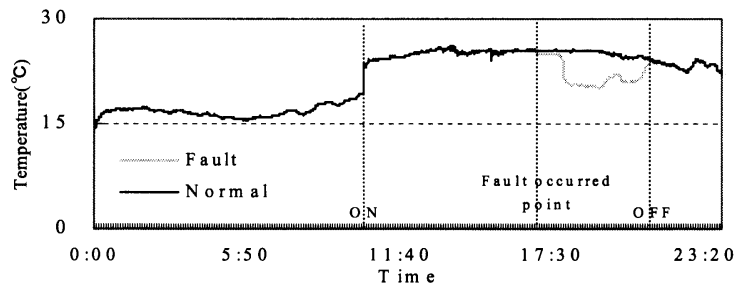

(a) Chilled water valve (response, room)

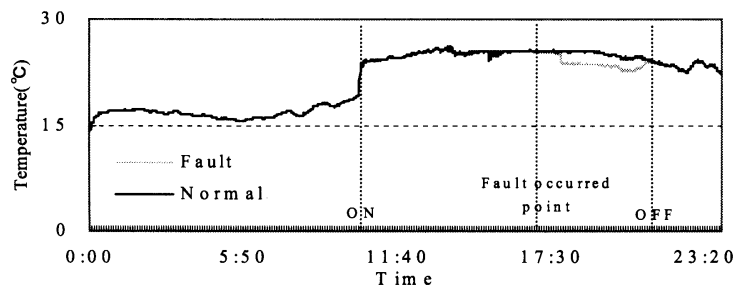

(b) Chilled water valve (stuck, room)

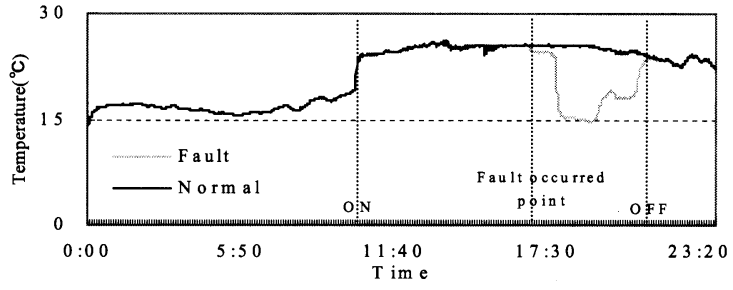

(c) Chilled water valve (full closed, room)

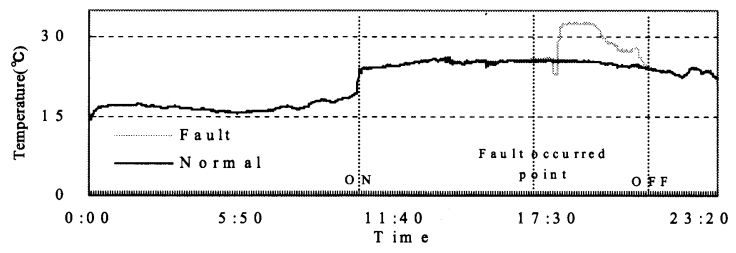

(d) Chilled water valve (full opened, room)

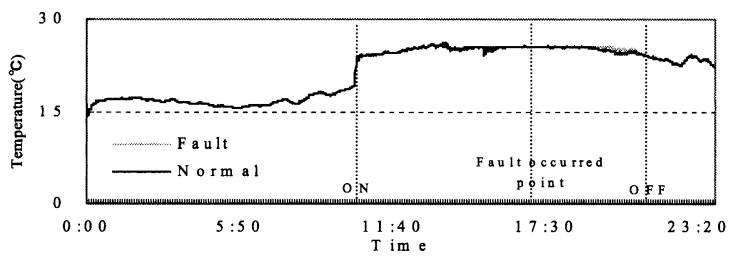

(e) Recycling air valve (full closed, room)

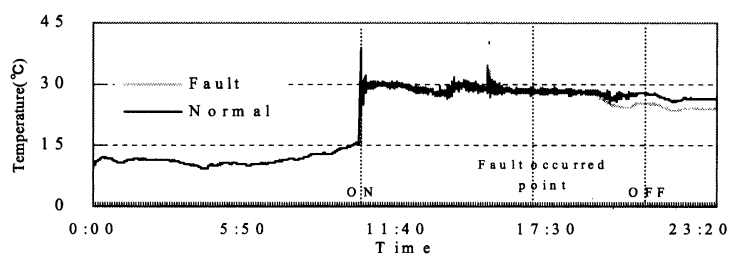

(f) Recycling air valve (full closed, heating unit entrance)

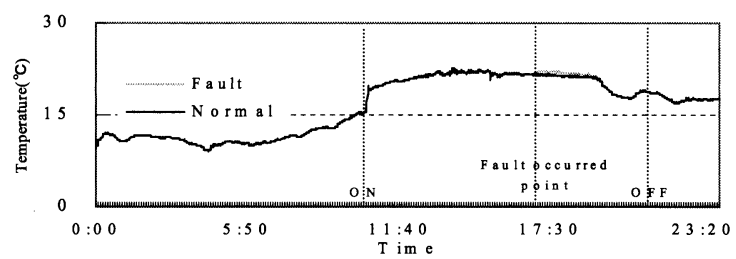

(g) Recycling air valve (full closed, heating unit exit)

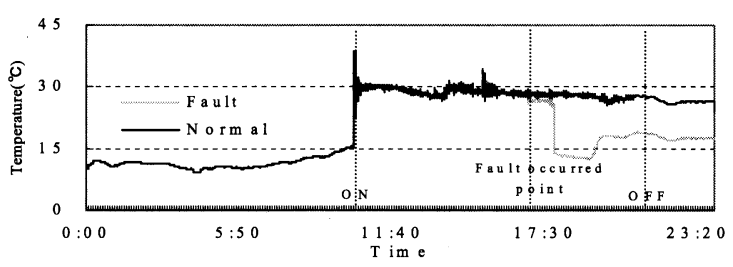

(h) Recycling air valve (full opened, heating unit entrance)

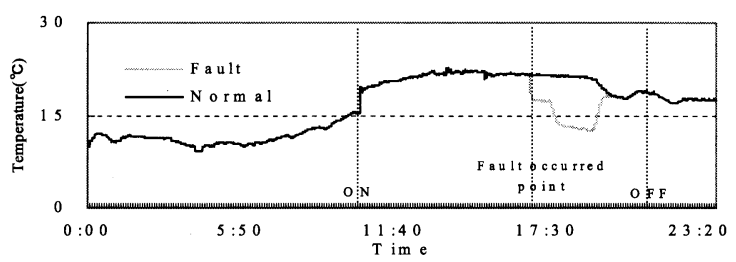

(i ) Recycling air valve (full opened, heating unit exit)

Fig. 5 Fault data samples. The valve condition of each fault and the temperature measuring point are shown in the bracket. 


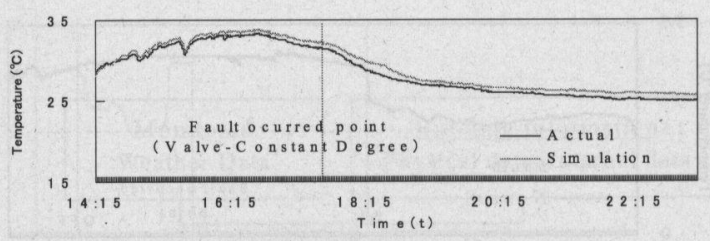

(a) Fault comparison case 1

Temperature at the time of fault occurrence

Simulator: hot water control valve, full-opened

Actual system : operation with the maximum capacity

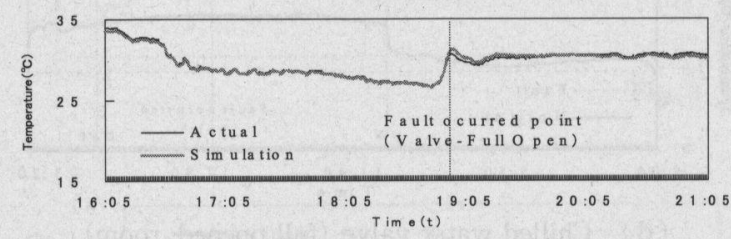

(b) Fault comparison case 2

Temperature at the time of fault occurrence

Simulator: hot water control valve, constantly opened

Actual system: operation with the minimum capacity

Fig.6 Comparison between simulation and actual data

opening degree of the valve is stuck at constant degree [Stuck] and the responsibility fault [Response] in which the valve opening degree is changed at every control with constant percentage of the correct opening degree. The setting temperature in the room was changed from $20^{\circ} \mathrm{C}$ to $28^{\circ} \mathrm{C}$ at the step of $1^{\circ} \mathrm{C}$. The faults were caused at various times from 8 a.m. to 6 p.m. every day. Some sample data of generated fault conditions are shown in Fig. 5 .

As a feasibility experiment, the authors compared the faults of the hot water volume control valve of the simulator with the data from the actual system when the valve was full-opened and constantly opened. In heating, the actual system operation with the maximum capacity and the minimum capacity represent the full opening and the constant opening of the valve, respectively. The results of the comparison are given in Fig. 6 ( a ) and ( b ). After the fault occurred, the room temperature of the simulator changed as the actual room temperature did.

\section{Fault Detection and Diagnosis Method}

\section{1 Recurrent Neural Network}

The authors examined the effectiveness of the RNNbased trend diagnosis method using data obtained from the above four fault conditions and those from normal conditions. Fig. 7 shows the composition of the three-layered RNN. Two things must be considered in determining the number of units in the hidden layer. On one hand, if the number of units is too small, the RNN does not have enough free parameters to approximate the underlying function well. On the other hand, if the number of units is

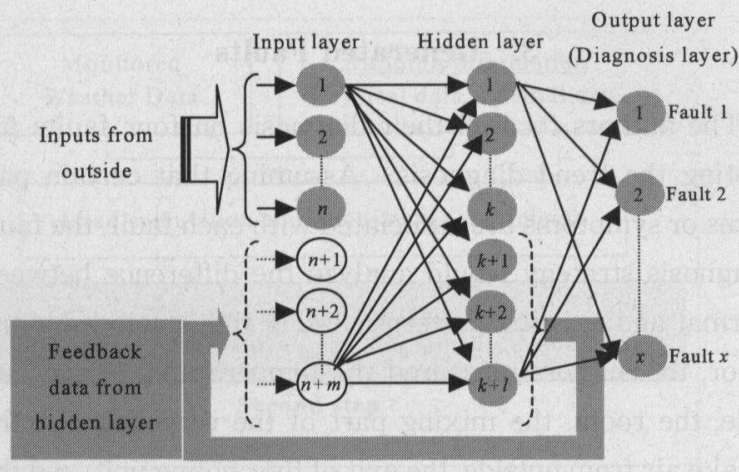

Fig. 7 RNN structure

too large, the model has too many free parameters and may loose its generalization ability. In this experiment, the number of the input units $n$ was 6 according to the number of defined inputs and the number of units in the output layer $x$ was 4 according to the number of faults. The output unit was allocated to each fault as shown in Fig. 7. We experimentally set the unit number in the hidden layer and feedback loop $m, k$ and $l$ as 5, 5 and 5, respectively.

\section{2 Training Algorithm}

Many training algorithms for RNN have been proposed up to now $^{8), 9)}$. The operation of the RNN is shown with the state equations of Eq. (20) and Eq.(21).

$$
\begin{aligned}
& \tau_{i} \frac{d x_{i}}{d t}=-x_{i}(t)+\sum_{j}^{N} w_{i j} y_{j}(t)+X_{i}(t) \\
& y_{i}=f\left(x_{i}\right)=\frac{1}{1+e^{-x_{i}}}
\end{aligned}
$$

In the above equations, $x_{i}, y_{i}$ and $X_{i}$ denote the internal state, the output and the input of the unit $i$ at time $t$, respectively; $w_{i j}$ is the weight between the unit $j$ and the unit $i$; $\tau_{i}$ is the delay coefficient in unit $i$. The number of units connected to the $i$-th unit is $\mathrm{N}$. The training of RNN is to reflect a part of all state vector $x(t)$ of the network in the time series parameters of the input vector $X(t)$ by adjusting weights and to convert the output vector $y(t)$ into the desired time series pattern. Here, the authors applied the finite time interval learning method, in which temporal dependency is not considered. The network operated for a certain period with fixed weight. After that, the weight was updated in the direction where the error between the output and the teacher signal decreases and reduces most of the learning error in given time period and makes the RNN's training efficiency. The well-known back propagation learning algorithm was used. The learning method is shown in Eq. (22).

$$
E(\boldsymbol{w}(n))=\int_{T_{1}}^{T_{2}} \sum_{k}^{N_{k}} \frac{1}{2}\left(y_{k}(t)-Y_{k}(t)\right)^{2} d t
$$


$E$ : Evaluation function of the learning while the input vector $X(t)$ sequence is applied from time $T_{1}$ to $T_{2}$.

$N_{k}$ : Number of units in the output layer.

$y_{k}(t)$ : Output of the unit $k$ in the output layer.

$Y_{k}(t)$ : Teaching signal of the unit $k$ in the output layer.

$\Delta w_{i j}$ is the change in $w_{i j}$ for one trial (the case $\Delta n=1$ ) and is shown in Eq. (23).

$$
\Delta w_{i j}=-\eta \frac{\partial E}{\partial w_{i j}}
$$

Then, the changes of $E$ is given in Eq. (24),

$$
\Delta E=\sum_{i, j} \frac{\partial E}{\partial w_{i j}} \Delta w_{i j}=-\eta \sum_{i, j}\left(\frac{\partial E}{\partial w_{i j}}\right)^{2}
$$

$\eta$ : Learning coefficient ( 0.7 in this study).

If $\Delta w_{i j}$ changes as Eq. (23), $E(w(n))$ decreases with an

Table 5 Input data

\begin{tabular}{l|l}
\hline \hline 1 & Outside air temperature \\
\hline 2 & Interior temperature \\
\hline 3 & Setting temperature \\
\hline 4 & $\begin{array}{l}\text { The temperature in the recycling air and the intake air } \\
\text { mixing part }\end{array}$ \\
\hline 5 & The temperature in the cooling unit exit \\
\hline 6 & The temperature in the heating unit exit \\
\hline
\end{tabular}

Table 6 Input data sets and the desired

\begin{tabular}{c|c|c|c|c|c}
\hline \hline \multirow{2}{*}{ Input date set } & \multicolumn{5}{|c}{ Desired output } \\
\cline { 2 - 6 } & Unit 1 & Unit 2 & Unit 3 & $\cdots \cdots$ & Unit $x$ \\
\hline Fault 1 & 1 & 0 & 0 & 0 & 0 \\
\hline Fault 2 & 0 & 1 & 0 & 0 & 0 \\
\hline Fault 3 & 0 & 0 & 1 & 0 & 0 \\
\hline$\cdots \cdots$ & 0 & 0 & 0 & 1 & 0 \\
\hline Fault $x$ & 0 & 0 & 0 & 0 & 1 \\
\hline Normal & 0 & 0 & 0 & 0 & 0 \\
\hline
\end{tabular}

increase in $n$.

The weights are updated according to Eq. (25),

$$
w(n+1)=w(n)+\Delta w(n)
$$

The database was divided into two parts: The first was for training (10 days) and the second was for testing (20 days). The input data are given in Table 5. All the weights and the thresholds were initialized randomly before training. All desired outputs were set to 0 for the normal condition data. For the fault condition data, 1 was given as the desired output of the output unit, which was allocated to each fault while the others were set to 0 . The relationship between the input data sets and the desired outputs are given in Table 6 . The training data-feeding rule is shown in Fig. 8. To confirm the fairness of the data feeding, we used a random selection rule of the training data.

The number of training terms for each fault and normal condition data was fixed to eliminate the bias.

\section{Fault Diagnosis Result}

Fig. 9 shows an example of the results of fault diagnosis of both data of fault condition (when fault occurred in the heating unit) and the normal conditions. For any fault condition data, the output of the unit to which a fault was allocated after it had occurred was near 1 , and the outputs of the other three units remained at almost 0 . For the normal condition data, the outputs of all the units remained at nearly 0 . The overall testing result for the normal data sets and the fault data sets are given in Table 7 and Table 8 respectively. The maximum, minimum and average values of the each output units were less than 0.5 for all normal data (Table 7) and over than 0.6

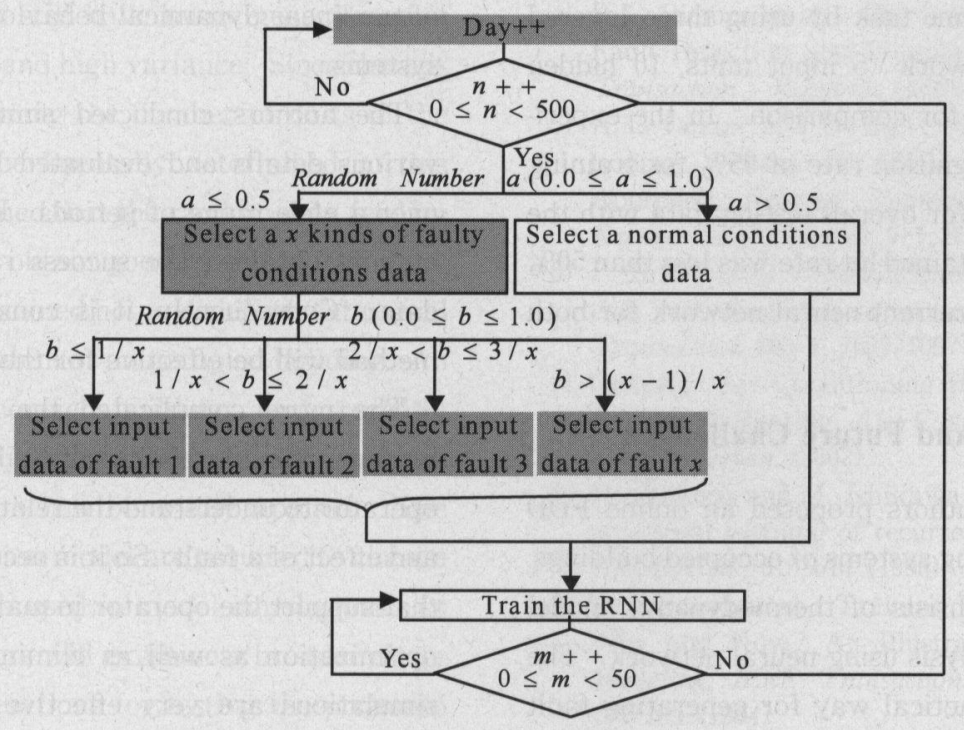

Fig. 8 Training data-feeding rule 


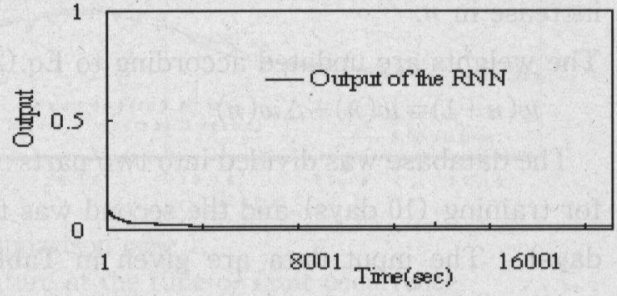

(a) Normal condition

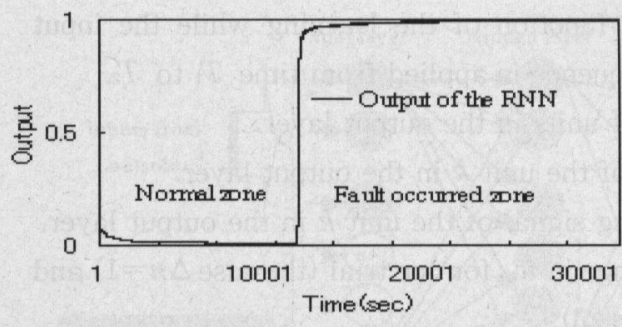

(b) Fault condition

Fig.9 Fault diagnosis sample

Table 7 Testing results for normal data

\begin{tabular}{c|c|c|c|c}
\hline \hline For correct data & Output Unit 1 & Output Unit 2 & Output Unit 3 & Output Unit 4 \\
\hline Maximum Value & 0.421 & 0.392 & 0.263 & 0.293 \\
\hline Minimum Value & 0.133 & 0.157 & 0.171 & 0.247 \\
\hline Average Value & 0.216 & 0.214 & 0.209 & 0.284 \\
\hline
\end{tabular}

Table 8 Testing results for faulty data

\begin{tabular}{c|c|c|c|c|c}
\hline \hline \multicolumn{2}{c|}{ For fault data } & Output Unit 1 & Output Unit 2 & Output Unit 3 & Output Unit 4 \\
\hline \multirow{4}{*}{ Cooling Unit } & Maximum Value & 1.000 & 0.243 & 0.339 & 0.426 \\
\cline { 2 - 5 } & Minimum Value & 0.737 & 0.192 & 0.248 & 0.218 \\
\cline { 2 - 5 } & Average Value & 0.839 & 0.211 & 0.295 & 0.369 \\
\hline \multirow{4}{*}{ Heating Unit } & Maximum Value & 0.411 & 1.000 & 0.387 & 0.308 \\
\cline { 2 - 5 } & Minimum Value & 0.235 & 0.726 & 0.273 & 0.237 \\
\cline { 2 - 5 } & Average Value & 0.306 & 0.877 & 0.351 & 0.289 \\
\hline \multirow{4}{*}{ Air intake Unit } & Maximum Value & 0.333 & 0.356 & 1.000 & 0.381 \\
\cline { 2 - 5 } & Minimum Value & 0.249 & 0.239 & 0.684 & 0.315 \\
\cline { 2 - 5 } & Average Value & 0.281 & 0.285 & 0.851 & 0.363 \\
\cline { 2 - 5 } & Maximum Value & 0.365 & 0.296 & 0.391 & 1.000 \\
\cline { 2 - 5 } & Avimum Value & 0.226 & 0.237 & 0.306 & 0.652 \\
\hline
\end{tabular}

for all fault data (Table 8). Considering these results, the threshold of the fault diagnosis can be set as 0.5. The authors also tested the same task by using three-layered non-recurrent neural network (5 input units, 10 hidden units and 4 output units) for comparison. In the experiment, we obtained a recognition rate of $95 \%$ for training data and more than $90 \%$ for overall testing data with the proposed method. The obtained hit rate was less than $50 \%$ when we used the non-recurrent neural network for both training and testing data.

\section{Conclusions and Future Challenges}

In this research, the authors proposed an online FDD method for air-conditioning systems of occupied buildings. The proposed method consists of thermodynamic model simulations and fault analysis using neural network. The model simulation is a practical way for generating fault and normal (fault-free) database, because it is not easy to obtain a variety of fault data from the actual systems. Another advantage of our study is to use RNN for analysis of nonlinear dynamical behaviors of the air-conditioning systems.

The authors conducted simulation experiments with various details and evaluated both heating and cooling over a wide range of periods. From the experiments, the authors obtained the success rate of over $90 \%$ for test data. Consequently, it is considered that the proposed method will be effective for the actual system.

The more complicated the building control systems become, the more difficult it becomes for an ordinary operator to understand the relationship between the cause and effect of a fault. So it is necessary to develop the tools that support the operator in making decisions for building optimization as well as eliminating faults. The model simulations are very effective for understanding those relationships. In this study, the authors developed a com- 
puter simulation model for understanding and obtaining the data of fault and normal conditions, instead of using an actual plant, because an actual plant is not available for obtaining the data of fault conditions.

Meanwhile, although several FDD methods have been proposed up to now, it is not easy to apply these FDD methods to an air-conditioning system considering their complexity and the nonlinear dynamic behavior.

Actually, the authors have not experimentally compared their method with other methods except feed forward neural network. Many of the other methods can be applied only on a component (equipment) base (e.g., physical models, polynomial models and AR type models) and require physical or mathematical knowledge of each component. They also require multiple parameter tunings. The hidden Markov models and the support vector machines can be also applied for FDD of the air-conditioning systems. But they are also mostly appropriate for component-base FDD to diagnose the component independently. The Markov models have no continuous internal states and the support vector machines have no internal states at all compared with the neural networks. Therefore, they are not suitable for continuous time series analysis. But the neural networks are black box models and the overall behavior of the air-conditioning system can be observed just by using input and output parameter mapping. In addition, the recurrent neural networks (RNN) can capture the hidden dynamics of nonlinear systems and trends in the time series data.

The number of units in the hidden layer is also important. If it is few, training errors and generalization errors will frequently occur due to under-fitting and high statistical bias. On the other hand, if it is too many, we may get fewer training errors but still have many generalization errors due to over-fitting and high variance. Since there is no strict rule for determining the number of units in the hidden layer, it was experimentally determined in this research. We also confirmed that the random data feeding rule is very important to obtain good results.

In this research, we examined hot and chilled water used in the air-conditioning system of an occupied building. An increasing number of occupied buildings have recently used this type of air-conditioning systems and the method the authors proposed here can be wildly applied. The parameters of the simulation model are required to change according to the buildings used in model simulations. In future research, the authors will examine a broader range of faults and different situations for testing the proposed method.
Finally, we would like to mention the model evaluation issue of complex systems like air-conditioning plant that we treated in this paper. It is not easy to prove the simulation model is enough for obtaining all the behavior data of the actual target system. In the Section 2.3 and the last part of the Section 3, we compared the simulation results with the behavior of the actual plant for some cases. Although it may not be enough for the diagnoses of the variety of faults, we consider that we can expect the fault in the actual plant is detectable if the fault case is clearly distinguishable from the normal case in simulation.

At present we have no effective method to evaluate quantitatively the model of the air conditioning system of building in use, which makes it difficult to discuss the total performances of the different FDD methods. This issue will be the final goal of our research.

\section{Acknowledgement}

This research was partially supported by the Grant-inAid for the WABOT-HOUSE Project by Gifu prefecture and the 21st Century of Excellence Programs, "The innovation research on symbiosis technologies for human and robots in the elderly dominant society" in Waseda University.

\section{References}

1) L. Haorong and E. B. James: An improved Method for Fault Detection and Diagnosis Applied to Packaged Air Conditioners, ASHRAE Transaction, 109-2, 683/692 (2003)

2) P. Carling: Comparison of Three Fault Detection Methods Based on Field Data of an Air-Handling Unit, ASHRAE Transaction, 108-1, 904/920 (2002)

3) S. R. Shaw : Detection and Diagnosis of HVAC Faults via Electrical Load Monitoring, International journal of Heating, Ventilating, Air-Conditioning and Refrigerating Research, 8-1, 13/40 (2002)

4) P. L. Riemer et al: : The Use of Time Series Analysis in Fault Detection and Diagnosis Methodologies, ASHRAE Transaction: Research (4589), 384/394 (2001)

5) A. L. Dexter and D. Ngo: Fault Diagnosis in Air-Conditioning System: A Multi-step Fuzzy Model-Based Approach, Heating, Ventilating, Air-Conditioning and Refrigerating Research, 7-1, 83/102 (2000)

6) P. House et al.: Classification techniques for fault detection and diagnosis of an air-handling unit, ASHRAE Transaction, 105-1, 1087/1097 (1999)

7) Ohmsha : Air-Conditioning Hand Book published by The Society of Heating, Air-Conditioning and Sanitary Engineers of Japan (2002)

8) H. Shimada and M. Ishikawa : Prediction of time series by structural learning of recurrent neural networks, Proc. of IIZUKA' 98, 610/614 (1998)

9) O. Nerrand et al.: Training Recurrent Neural Networks : Why and How? An Illustration in Dynamical Process Modeling, IEEE Transactions on Neural Networks, 5-2, 178/183 (1994) 


\section{Appendix}

\section{Nomenclature}

$A M F_{F}$ : Endothermic correction factor for illumination $A_{w \_d}\left[\mathrm{~m}^{2}\right]$ : Area of the wall on direction $d$ $A_{g_{-} d}\left[\mathrm{~m}^{2}\right]$ : Area of the glass on direction $d$ $A_{\text {roof }}\left[\mathrm{m}^{2}\right]$ : Area of the roof

$A_{\text {floor }}\left[\mathrm{m}^{2}\right]$ : Area of the floor

$C C_{R}$ : Convection component of the solar radiation acquisition

$d$ : Direction (N, NE, E, EW, W, WS, S, SN, N)

$K_{w_{-} d}\left[\mathrm{~J} / \mathrm{m}^{2} \cdot \mathrm{K} \cdot \mathrm{sec}\right]$ : Overall heat transfer coefficient of the wall on direction $d$

$K_{g_{-} d}\left[\mathrm{~J} / \mathrm{m}^{2} \cdot \mathrm{K} \cdot \mathrm{sec}\right]$ : Heat transfer coefficient of the glass on direction $d$

$K_{\text {roof }}\left[\mathrm{J} / \mathrm{m}^{2} \cdot \mathrm{K} \cdot \mathrm{sec}\right]$ : Heat transfer coefficient of the roof $K_{\text {floor }}\left[\mathrm{J} / \mathrm{m}^{2} \cdot \mathrm{K} \cdot \mathrm{sec}\right]$ : Heat transfer coefficient of the floor $k_{d}$ : Direction factor

$K_{H V}$ : Standard specific heat (air)/Standard specific volume (air)

$K_{\text {WELH }}$ : Evaporation latent heat (water)/Standard specific volume (air)

$Q_{D}[J]$ : Heat of the drafts

$q_{D_{-} S}[\mathrm{~J}], q_{D_{-} L}[\mathrm{~J}]$ : Sensible and latent heat of the drafts respectively

$Q_{G_{-} \text {out }}[\mathrm{J}]$ : Heat transfer through outer glass windows

$Q_{G_{-} \text {in }}[J]$ : Heat transfer through inner glass windows

$q_{G C_{-} d}[\mathrm{~J}]$ : Percolation heat of the glass windows

$q_{G R_{-} d}[\mathrm{~J}]$ : Solar radiation heat of the glass windows

$Q_{H}[\mathrm{~J}]$ : Heat from the human body

$q_{H L}[\mathrm{~J}], q_{H S}[\mathrm{~J}]$ : Latent heat and the sensible heat from the human body respectively

$Q_{I}[\mathrm{~J}]$ : Heat from the illumination

$q_{I F}[\mathrm{~J}], q_{I I}[\mathrm{~J}]$ : Heat from the fluorescent bulbs and incandescent bulbs respectively

$Q_{\text {roof }}[\mathrm{J}]$ : Heat transfer through roofs

$Q_{\text {floor }}[\mathrm{J}]$ : Heat transfer through floors

$Q_{W_{-} \text {in }}[J]$ : Heat transfer through inner walls

$Q_{W_{-} \text {out }}[\mathrm{J}]$ : Heat transfer through outer walls

SCT : Sheltering coefficient
$S S G\left[\mathrm{~J} / \mathrm{m}^{2} \cdot \mathrm{sec}\right]$ : Standard solar radiation acquisition of the glass

$S_{G R_{-} d}\left[\mathrm{~J} / \mathrm{m}^{2} \cdot \mathrm{sec}\right]$ : Standard solar radiation acquisition of the glass on direction $d$

$S_{G R P_{-} d}\left[\mathrm{~J} \cdot \mathrm{m}^{2} \cdot \mathrm{sec}\right]$ : Peek of the standard solar radiation acquisition on direction $d$

$S_{G R L_{d} d}$ : Late factor of solar radiation load

$S C_{d}$ : Sheltering coefficient on direction $d$

$T_{\text {out_d } d}\left[{ }^{\circ} \mathrm{C}\right]$ : Outer air temperature on direction $d$

$T_{\text {room }}\left[{ }^{\circ} \mathrm{C}\right]$ : Interior air temperature

$T_{\text {out_roof }}\left[{ }^{\circ} \mathrm{C}\right]$ : Air temperature at outside of the roof

$T_{\text {out_floor }}\left[{ }^{\circ} \mathrm{C}\right]$ : Air temperature at outside of the floor

$T_{\text {nroom_d }}\left[{ }^{\circ} \mathrm{C}\right]$ : Interior temperature of the next room on direction $d$

$T_{\text {out_av }}\left[{ }^{\circ} \mathrm{C}\right]$ : Average Outer air temperature

$V_{D}\left[\mathrm{~m}^{3}\right]$ : Draft

$W_{F}, W_{I}\left(\left[\mathrm{~J} / \mathrm{m}^{2} \cdot \mathrm{sec}\right]\right)$ : Wattage (fluorescent valve), Wattage (incandescent valve)

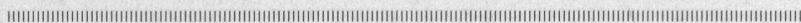

\section{Upali Samarasinghe Herath Kumarage (Member)}

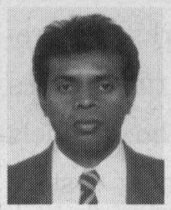

He received the B.E. and M.E. degrees from the Waseda University in 1994 and 1996 respectively. He is currently working as a visiting research fellow at the Advanced Research Institute for Science and Engineering of Waseda University and a visiting lecture at the Shukutoku University in Saitama. He is mainly involved in the research of neural networks. He has been applied the neural networks for prediction problems, controlling problems and fault detection and diagnosis problems etc.

\section{Shuji Hashimoto (Member)}

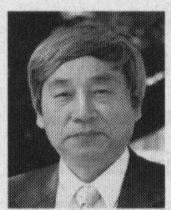

He received the B.S. and Dr. Eng degrees in Applied Physics from Waseda University in 1970 and 1977 , respectively. He is currently a professor at the Department of Applied Physics, Waseda University. His research interests include neural networks, image processing, humanoid robots, and Kansei information processing. 